\title{
Synthesis of Log-Domain Filters from First-Order Building Blocks
}

\section{R. TIMOTHY EDWARDS AND GERT CAUWENBERGHS}

Electrical and Computer Engineering, Johns Hopkins University, Baltimore, MD 21218-2686

E-mail:tim@bach.ece.jhu.edu,gert@bach.ece.jhu.edu

\author{
Accepted August 31, 1998
}

\begin{abstract}
Log-domain filters are an important class of current-mode circuits having large-signal linearity and increased tuning range over voltage-mode filter circuits of similar complexity. In this paper we describe synthesis of a single-ended, first-order filter circuit from static and dynamic translinear circuit principles, and show how higher-order filters can be easily constructed from the first-order building block. We address additional issues related to low-frequency (audio-frequency) filter design and present results measured from test circuits and a complete 15-channel filterbank system fabricated in $2 \mu \mathrm{m}$ and $1.2 \mu \mathrm{m}$ BiCMOS processes.
\end{abstract}

Key Words: log-domain filters, filterbanks, current-mode analog VLSI

\section{Introduction}

Log-domain filters comprise a subclass of circuits having externally linear transfer functions but internally nonlinear components $[1,2]$. As the name implies, log-domain filters are specifically those circuits whose internal state is a logarithmic function of the input and output. The circuit design exploits this particular nonlinearity directly rather than attempting linearization around an operating point. The result is that log-domain filters have large-signal linearity: the transfer function describes the overall behavior of the system. The equations governing the internal nonlinearity of the system are generally tractable, leading to complete solutions which do not require separate DC and transient analyses.

Substantial progress has been made in simplifying the processes of synthesis and analysis [3-8] by recognizing that log-domain filters are by necessity composed of translinear circuits, and using translinear circuit theory to avoid explicitly solving transistor $I-V$ equations. A crucial step in simplifying log-domain synthesis and analysis is to formulate a translinear principle which applies to time-derivative systems. This "dynamic translinear principle", was developed in Mulder et al. [4], and is worth paraphrasing here in the context of our method of circuit synthesis, where it allows us to synthesize filter circuits without resorting to state-space manipulation (cf. [5]).

\section{Principles of Log-Domain Design}

Translinear loop circuits, introduced by Gilbert [9,10], are a fundamental structure of log-domain circuits. Fig. 1 shows an example of a translinear loop formed by the base-emitter junctions on bipolar transistors. For purposes of analysis and synthesis, we will consider all transistors (whether BJT or, equivalently, MOSFET operating in weak inversion) to be ideal, perfectly matched devices implementing the simplified exponential function $I_{c}=I_{s} e^{V_{b e} / V_{t}}$, or inversely, $V_{b e}=V_{t} \ln \left(I_{c} / I_{s}\right)$, where $V_{b e}$ is the BJT base-toemitter voltage, or the gate-to-source voltage of a corresponding subthreshold MOS transistor, $V_{t}$ is the thermal voltage $\left(0.025 \mathrm{~V}\right.$ at room temperature) and $I_{s}$ is the reverse saturation current of the transistor. Circuit modifications necessary to deal with the nonidealities of real devices will be addressed in Section 5.

By Kirchoff's voltage law, the sum of the $V_{b e}$ voltages around the loop is zero:

$$
\sum_{i \text { odd }} V_{b e_{i}}=\sum_{i \text { even }} V_{b e_{i}}
$$

Substituting the idealized transistor equation yields the "static translinear principle": 


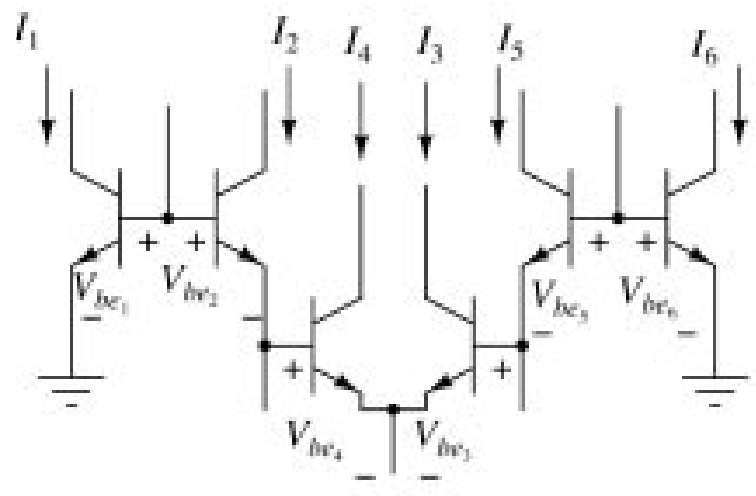

Fig. 1. Translinear loop with common-base and common-emitter configurations.

$$
\begin{aligned}
\sum_{i \text { odd }} V_{t} \ln \left(I_{i} / I_{s}\right) & =\sum_{i \text { even }} V_{t} \ln \left(I_{i} / I_{s}\right) \\
\prod_{i \text { odd }} I_{i} & =\prod_{i \text { even }} I_{i}
\end{aligned}
$$

Just as the static translinear principle gives a simple rule for computing products of currents, we need a simple rule to generate equations involving time-derivatives of currents, i.e.

$$
I_{\text {out }} \propto \dot{I}_{\text {in }}
$$

The property of the derivative of an exponential function is the key to this problem. Applying the derivative to the simplified transistor equation results in the "dynamic translinear principle":

$$
\begin{aligned}
I_{\text {out }} & =I_{s} e^{\left(V_{b e}\right) / V_{t}} \\
\dot{I}_{\text {out }} & =\frac{\mathrm{d}}{\mathrm{d} t}\left(I_{s} e^{\left(V_{b}-V_{e}\right) / V_{t}}\right) \\
& =\frac{1}{V_{t}} I_{\text {out }} \frac{\mathrm{d}}{\mathrm{d} t}\left(V_{b}-V_{e}\right)
\end{aligned}
$$

Grounding the BJT emitter $\left(V_{e}=0\right)$ and adding a capacitor $C$ to the system $\left(I_{C}=C \dot{V}_{b}\right)$ yields an equation composed entirely of current-mode variables. Fig. 2 shows such a system, a basic buildingblock of log-domain filters (cf. [3], Fig. 1, and [4], Fig. 1 ), in which a constant voltage $V_{\text {shift }}$ may be inserted between the capacitor node $Z$ and the transistor base without affecting the equation solution:

$$
\dot{I}_{\text {out }}=\frac{1}{V_{t}} I_{\text {out }} \frac{I_{C}}{C}
$$

From this equation we can compute the derivative of a current by multiplying two currents together,

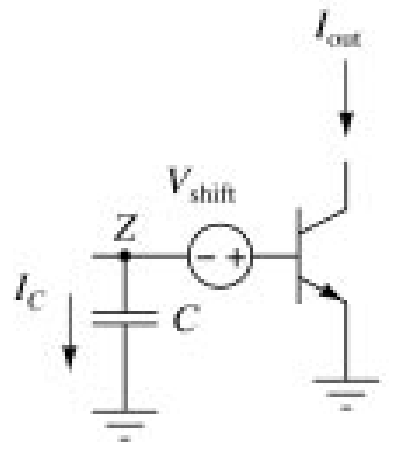

Fig. 2. Filter pole formed using a transconductor.

which can be done using a translinear loop circuit. Note that both equations (3) and (8) describe circuit behavior without explicitly referring to either exponent and logarithm functions or to device parameters (e.g. $I_{s}$ ).

\section{First-Order Circuit Synthesis}

To show how these circuit concepts can be used in practice to synthesize complete filter circuits, consider for instance a generic first-order system with the (current) transfer function:

$$
\frac{I_{\text {out }}(s)}{I_{\text {in }}(s)}=\frac{1}{A+\tau s}
$$

Equation (9), which is not in a canonical form, is a useful building block for generating higher-order circuits, as we will show in Section 4. Returning to the time domain, and substituting equation (8):

$$
\begin{aligned}
A I_{\text {out }}+\left(\frac{\tau I_{C}}{V_{t} C}\right) I_{\text {out }} & =I_{\text {in }} \\
I_{\text {out }}\left(A+\frac{\tau I_{C}}{V_{t} C}\right) & =I_{\text {in }}
\end{aligned}
$$

We then can define the time constant $\tau$ in terms of some (constant) bias voltage $I_{b}$ :

$$
\tau=\frac{V_{t} C}{I_{b}}
$$

Substituting $\tau$ into equation (11) and multiplying through by $I_{b}$, we get

$$
I_{\text {out }}\left(A I_{b}+I_{C}\right)=I_{\text {in }} I_{b}
$$

Equation (3) is a four-component translinear loop 
equation, with the constraint that $I_{C}$ and $I_{\text {out }}$ must have the relationship shown in Fig. 2. One of several possible implementations is the "up-down", (common-base) configuration shown in Fig. 3, in which transistors $Q 1$ through $Q 4$ form the translinear loop $I_{\text {out }} I_{1}=I_{\text {in }} I_{b}$. Q3 is a voltage level-shifter (cf. [3], Fig. 2), and equation (9) is satisfied if $I_{a}=I_{b}(1+A)$. The power supply, $V_{s s}$, is kept at about $-0.5 \mathrm{~V}$ to keep the current sources operating. The entire circuit is biased by adding a positive DC current to the input to keep the transistor base-emitter junctions forward biased.

\section{Designing Second-Order Sections}

Construction of higher-order log-domain circuits from simple component cells has been described in various ways, such as the Bernoulli cell of [5] and the E+ and E- cells of [8]. We generate higher-order functions in a similar way, factoring the desired current transfer function into equations which can directly be implemented by the first-order subcircuit of Fig. 3 . Consider, for example, the second-order bandpass equation [3]

$I_{\text {out }}(s)=I_{\text {in }}(s) \frac{\tau s}{1+(1 / Q) \tau s+\tau^{2} s^{2}}+I_{\mathrm{DC}}(s)$

in which the constant current $I_{\mathrm{DC}}$, independent of the signal, is added to the output of the system. This

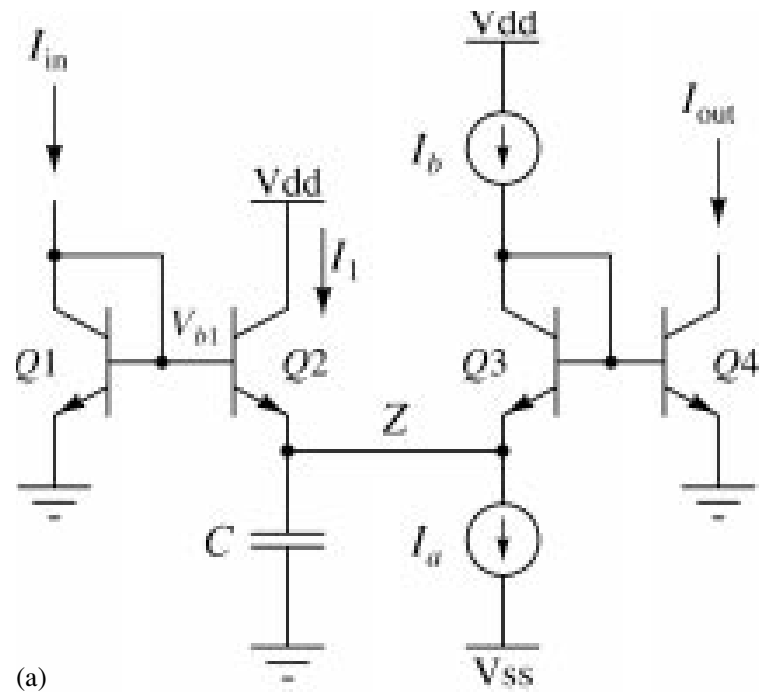

Fig. 3. First-order log-domain filter circuit. makes the output unidirectional, a form convenient for cascading filter stages. Equation (14) can be factored into two simple first-order current transfer functions by introducing an intermediate current term $I_{x}$ :

$$
\begin{aligned}
\frac{I_{\mathrm{out}}(s)}{I_{\mathrm{in}}(s)-I_{x}(s)} & =\frac{1}{1 / Q+\tau s} \\
\frac{I_{x}(s)}{I_{\mathrm{out}}(s)-I_{\mathrm{DC}}(s)} & =\frac{1}{\tau s}
\end{aligned}
$$

The right-hand side of (15) and (16) takes the form of the first-order function (9): for the first section, a lossy integrator, set $I_{a}=I_{b}(1+1 / Q)$, and for the second section, a lossless integrator, set $I_{a}=I_{b}$. The input to each section, however, has a negative feedback term from the other section, which cannot be directly subtracted from the system input without introducing negative currents. This situation, shown in Fig. 4(a), would drive the transistors out of the active mode and invalidate the translinear model. Instead, with the proper transformation, the negative feedback term can be referred to the capacitor node as a dependent current source, as shown in Fig. 4(b). The translinear loop equations for circuits $\mathrm{A}$ and $\mathrm{B}$ are

$$
\begin{aligned}
\left(I_{\mathrm{in}}-I_{z}\right) I_{b} & =\left(I_{a}+I_{C}-I_{b}\right) I_{\mathrm{out}} ; \\
I_{\mathrm{in}} I_{b} & =\left(I_{z}^{\prime}+I_{a}+I_{C}-I_{b}\right) I_{\mathrm{out}}
\end{aligned}
$$

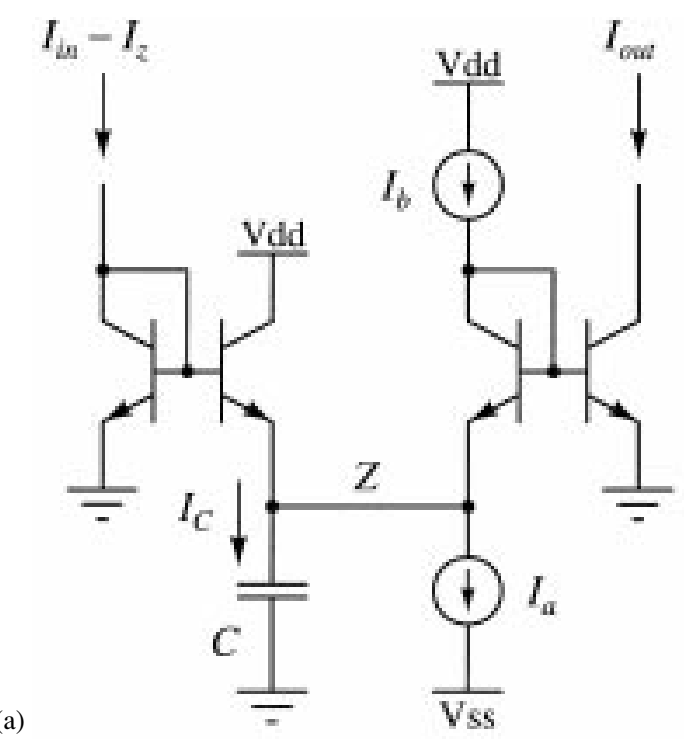

Fig. 4. Computing a current difference at a log-domain filter input. (a) The underlying idea, which is physically unrealizable. (b) An equivalent working implementation. 


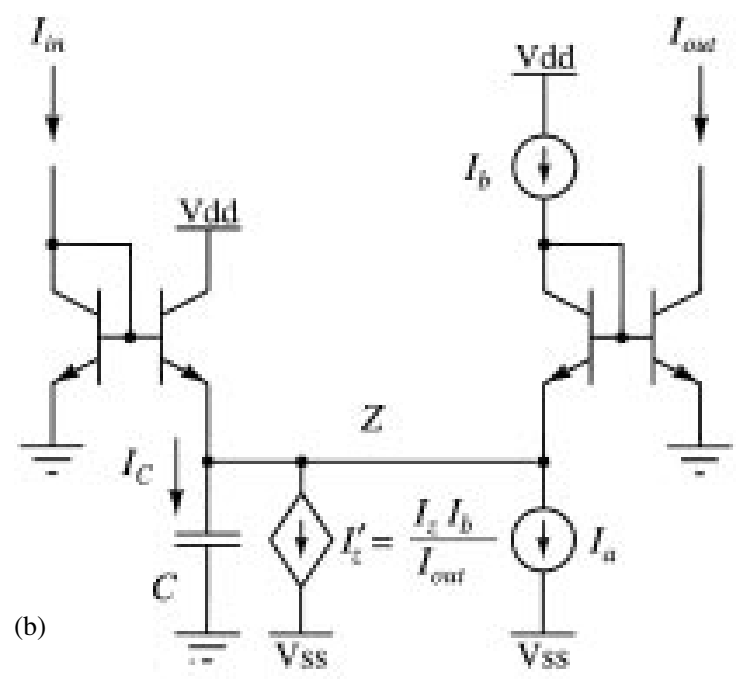

Fig. 4. (Continued)

Solving for $I_{z}^{\prime}$, we find that $I_{z}^{\prime}=\left(I_{z} I_{b}\right) / I_{\text {out }}$, a form which can be conveniently implemented by a translinear loop circuit.

Now the bandpass filter can be drawn as a cascade of two first-order circuits, with the output of the first section shared with the input of the second, as shown in Fig. 5. By noting the translinear loop $Q 2-Q 3-Q 4-$ $Q 5$ which gives the equation $I_{x} I_{y}=I_{b} I_{\text {out }}$, the dependent current sources $I_{\mathrm{DC}}^{\prime}$ and $I_{x}^{\prime}$ can be written in terms of $I_{y}$ rather than $I_{x}$, and the entire subcircuit which generates $I_{x}$ (dotted box in Fig. 5) can be eliminated, avoiding the need for one set of matched current sources.

It remains to generate the translinear loops implementing the dependent current sources. Two different solutions are illustrated in the bandpass circuit schematic of Fig. 6: In the first solution, $I_{x}^{\prime}=I_{b}^{2} / I_{y}$ is generated by the internal translinear loop $Q 3-Q 7-Q 6-Q 1$ using the common-emitter configuration of transistors $Q 6$ and $Q 7$. This configuration has been used by Frey [3] and others. The other dependent current source, $I_{\mathrm{DC}}^{\prime}=I_{\mathrm{DC}} I_{y} / I_{\text {out }}$, is implemented through the translinear loop $Q 8-Q 9-Q 3-Q 2$ using a common-base instead of a common-emitter configuration, in which the sign of the current is reversed, as required in Fig. 5, by mirroring, doubling, and subtracting from the same node. This configuration has been described previously by Fox [8]. Notice that both translinear loops implement the same function with different circuits. In each of the works mentioned above, the same circuit configuration (common-base or common-emitter) was used twice. We use both methods in the same filter, for the following reasons: On the left-hand side of the filter, a common-base configuration is generally less desirable due to the required extra pair of matched $I_{b}$ current sources. This circuit also incurs a signal delay around the current

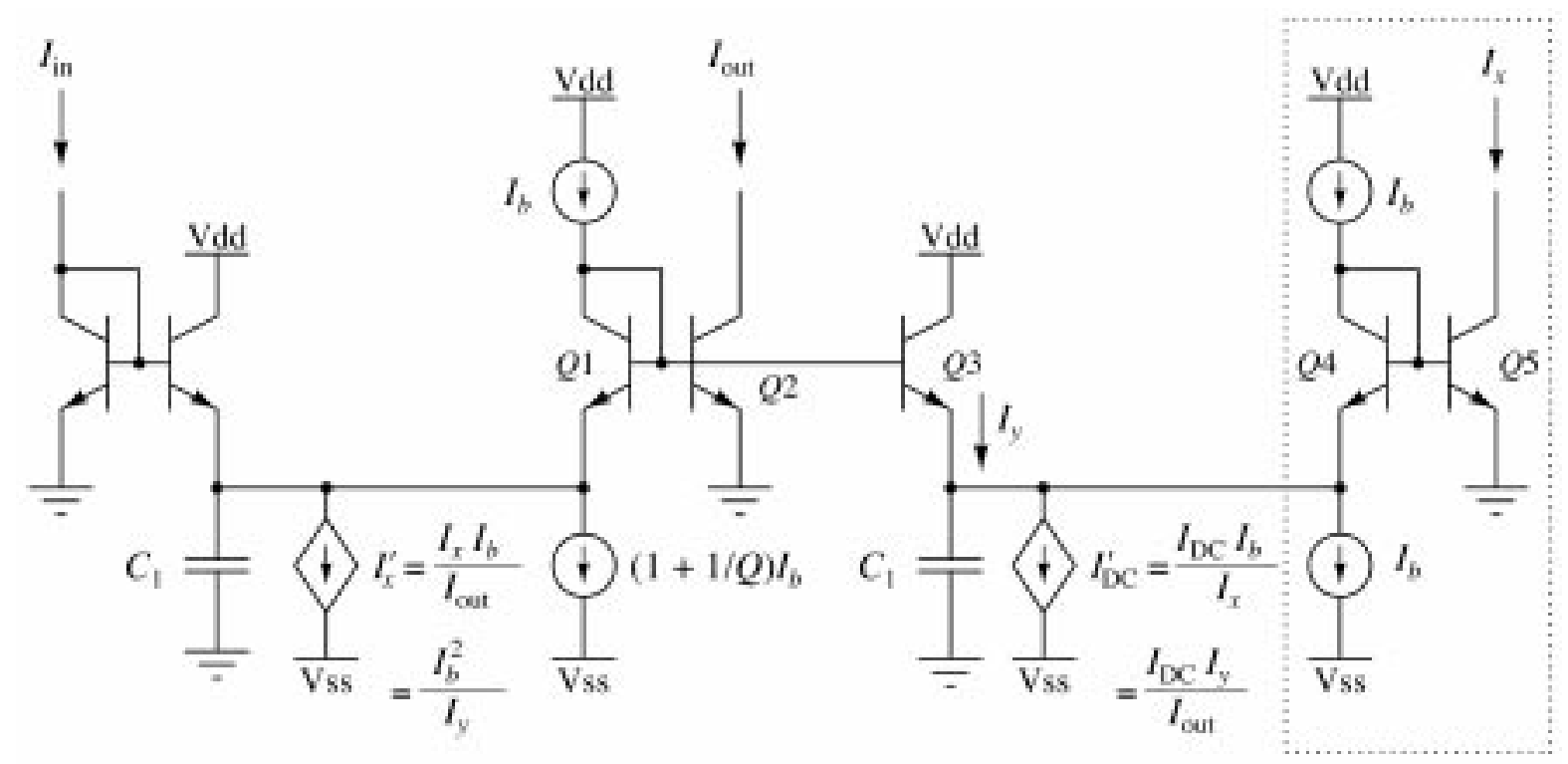

Fig. 5. Bandpass structure formed from first-order sections. 


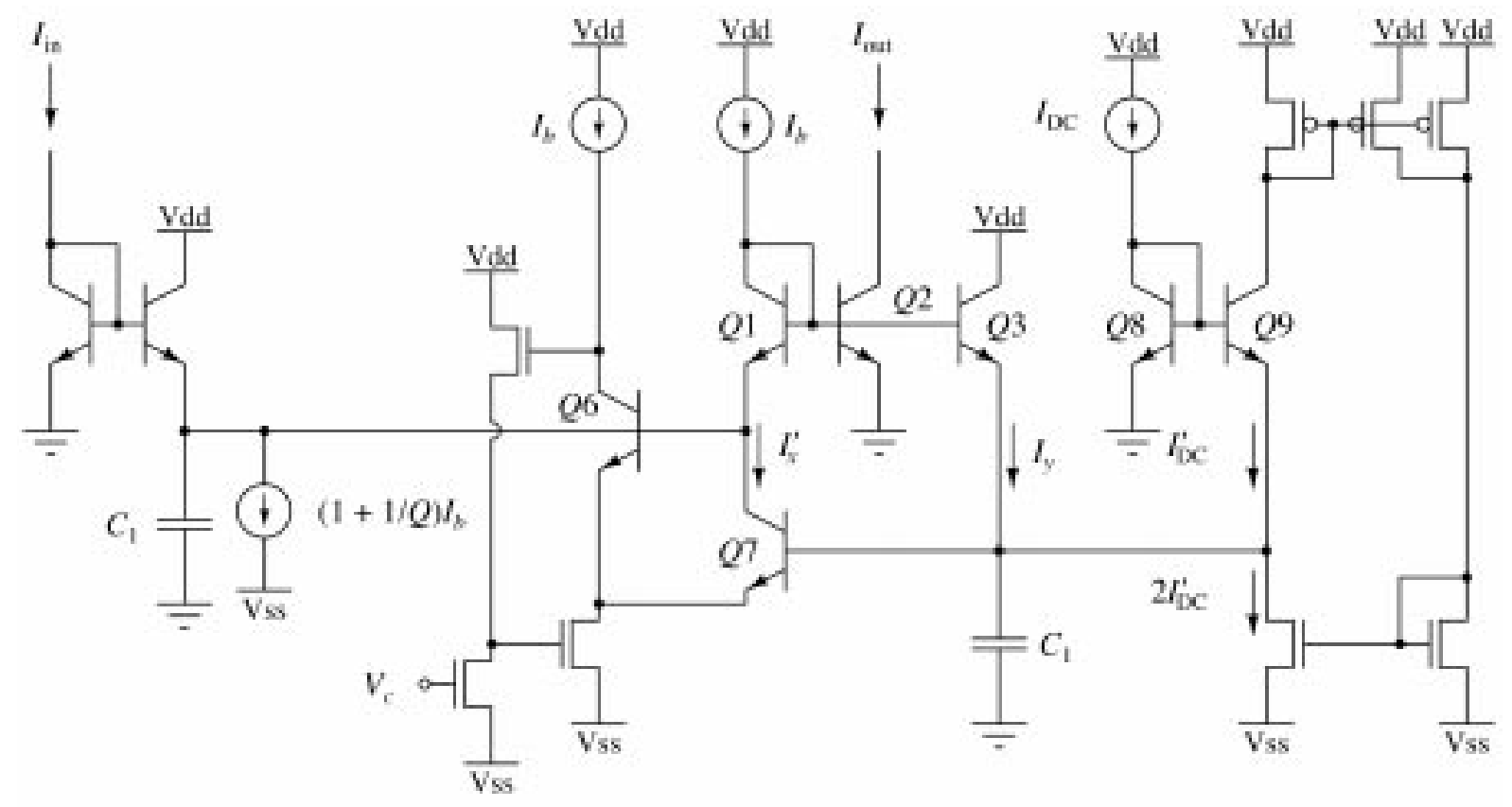

Fig. 6. Simplified circuit schematic for the second-order bandpass filter.

mirror loop, and has been observed to latch up when the MOSFETs in the mirrors are made too large, increasing the signal delay to the point at which the circuit becomes unstable. A full analysis of this circuit's stability has not been attempted.

On the right-hand side of the filter, use of a common-emitter circuit to replace the $Q 8-Q 9$ common-base pair (Fig. 7, with $V_{b 7}=0 \mathrm{~V}$ ) results in current draw through the base of $Q 6$, turning the lossless integrator into a lossy integrator. At audio frequencies, where the bias current $I_{b}$ can be smaller than the base current $I_{\mathrm{DC}} / \beta$ of 10 to $100 \mathrm{nA}$, the circuit will fail. Note, however, that $I_{\mathrm{DC}}^{\prime}$ is proportional to $I_{\mathrm{DC}} \cdot e^{V_{b 7} / V_{t}} \cdot I_{\mathrm{DC}}$ can be reduced to the order of magnitude of $I_{b}$ if $V_{b 7}$ is raised to compensate. This will allow the common-emitter circuit to work, though with the drawback that the output bias level is no longer equal to $I_{\mathrm{DC}}$, but is a derived function of $I_{\mathrm{DC}}$ and $V_{b 7}$.

\section{Technology Limitations for Low-Frequency Filter Design}

Much previously published work on log-domain filters has extolled their potential for replacing

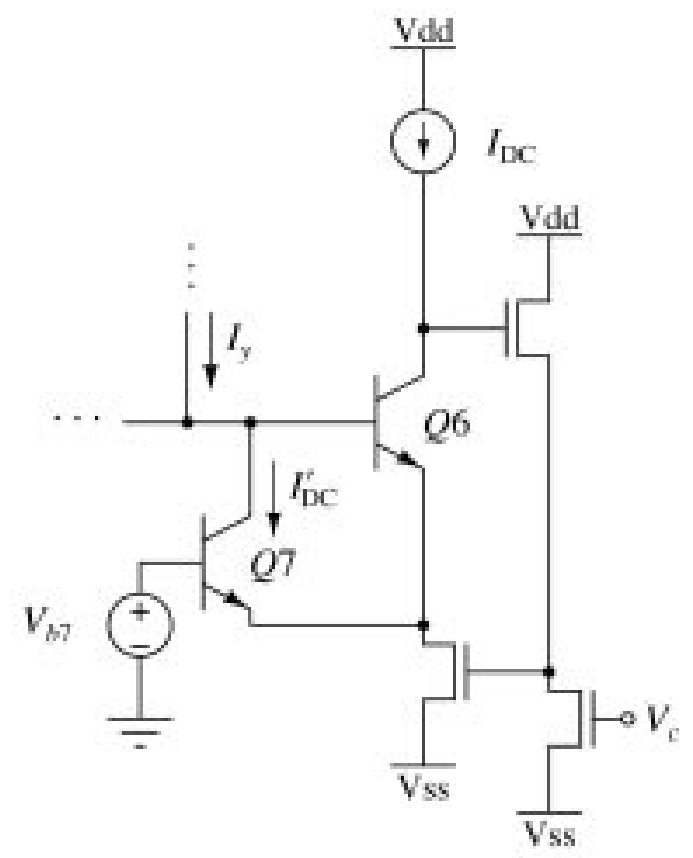

Fig. 7. An alternative common-emitter circuit generating $I_{\mathrm{DC}}^{\prime}$ (see text for discussion). 
conventional filters in high-frequency filter design. Our work instead concentrates on the use of logdomain filters for audio-frequency applications $[8,11,12]$, the use of current-mode filtering for current-mode applications, and the application of log-domain filters in system design [13]. The low frequency range of audio requires a large $\mathrm{RC}$ time constant, which for the log-domain filters described here is inversely proportional to the bias current $I_{b}$. For large-scale integrated systems where capacitors cannot reasonably be made larger than a few picofarads, the bias current can be as low as several tens of picoamps on the low end of the audio frequency range, which places some important restrictions on circuit technology. Problems arising from established designs are:

1. Traditional bipolar designs fail due to base current draw and can suffer from $\beta$ mismatch at low emitter current values.

2. MOSFET designs have poor matching of currents in mirrors, and require that the input bias current be very low to keep the MOSFET transistors in weak inversion. The input and output are then close to the noise floor and it

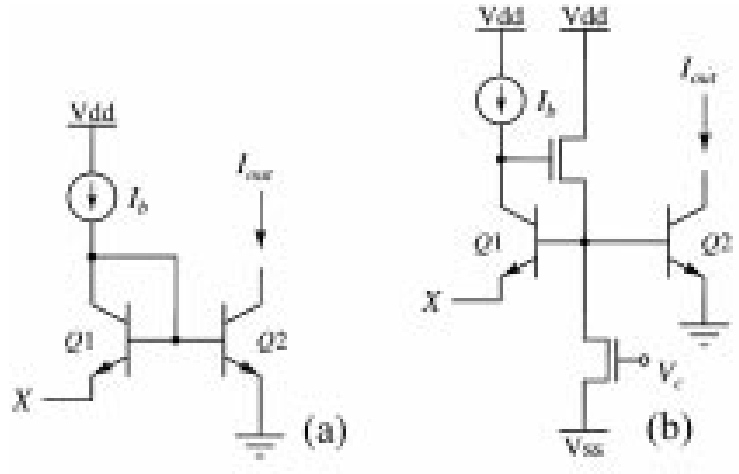

Fig. 8. Base compensation (b) eliminates undesirable behavior due to significant base current which occurs in (a).

becomes difficult to retrieve a clean output signal.

Having fabricated and tested both MOSFET and BiCMOS designs, we determined that the first of these two problems was probably the easiest to overcome. Base current loss can be eliminated using base-current compensation on all diode-connected transistors in the translinear loops as shown in Fig. 8. Voltage $V_{c}$ is a constant bias which reduces the impedence of the base

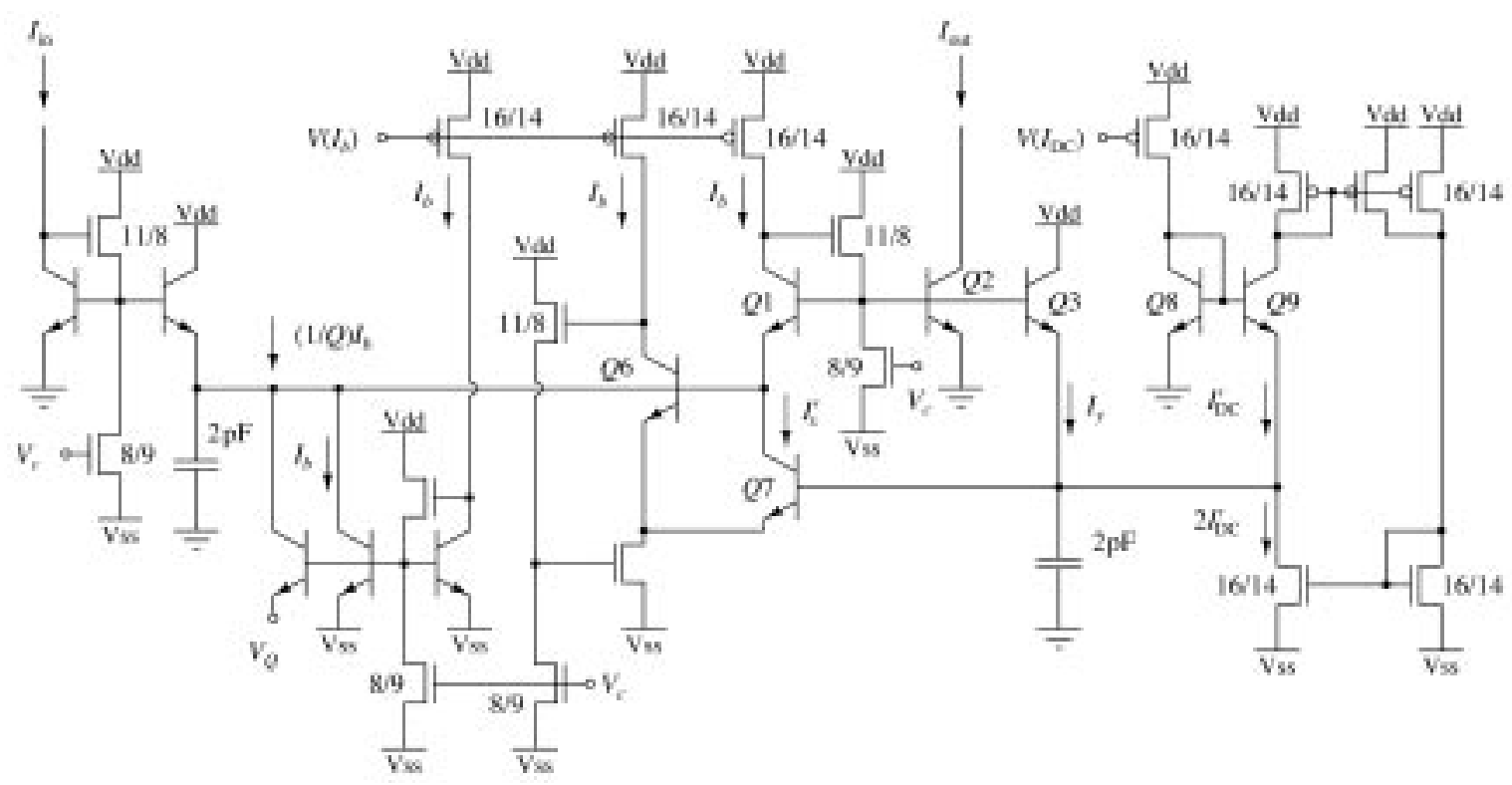

Fig. 9. Complete circuit schematic for the second-order bandpass filter. Bipolar transistors are minimum size, and MOS dimensions are indicated as $W / L$ in units of $\lambda=0.6 \mu \mathrm{m}$. 


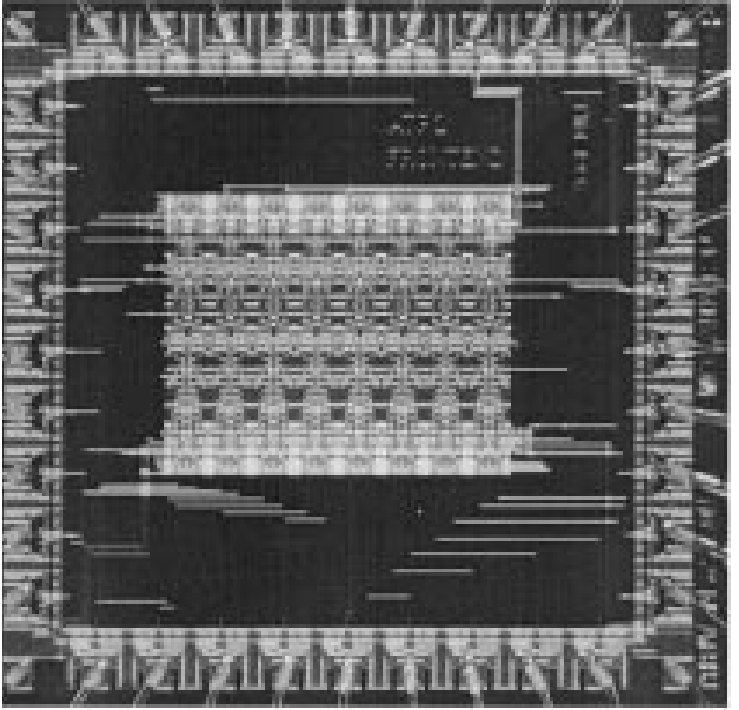

Fig. 10. Photograph of the fifteen-channel bandpass filterbank fabricated in $1.2 \mu \mathrm{m}$ technology inside a $2.2 \mathrm{~mm} \times 2.2 \mathrm{~mm}$ padframe.

node, and in the second-order filter can be shared with the $V_{c}$ bias in Fig. 6.

Fig. 9 shows the complete bandpass filter, including all base compensation circuits and current sources, as it was fabricated and from which the measurements in Section 6 were taken. Note the circuit implementation of the current $(1+1 / Q) I_{b}$ : in this configuration, $Q=e^{\left(V_{Q}-V_{s s}\right) / V_{t}}$, making tuning of $Q$ independent of $I_{b}$, the center frequency tuning control, and giving $Q$ a natural range from 1 to the practical maximum allowed by the circuit.

\section{Experimental Results}

We have fabricated and tested several different chips, one of which was a test chip with individual filter structures on it, and one a complete signal-processing system [13] containing a filterbank of log-domain bandpass filters (Fig. 10). The filterbank consists of 15 channels each consisting of two cascaded bandpass filters followed by rectification and smoothing of the signal at the output; this filterbank is used as an acoustic frontend for a simple template-based signal classifier. The classifier circuit is a current-mode system which takes the current outputs of the frontend system directly, without converting to voltage. The chips were fabricated on $2 \mathrm{~mm}$ dies in $2 \mu \mathrm{m}$ (test chip) and $1.2 \mu \mathrm{m}$ (filterbank) analog $n$-well BiCMOS processes, with double metal, double poly, and a $p$ base layer for creating vertical NPN bipolar transistors. For high-density integration, we limited capacitors to about $2 \mathrm{pF}$ and made all BJTs with a minimum size well. The size of the capacitors implies (from equation (12)) bias currents of $30 \mathrm{pA}$ to $3 \mathrm{nA}$ for center frequencies spaced from $100 \mathrm{~Hz}$ to $10,000 \mathrm{~Hz}$, respectively.

Fig. 11 shows measurements taken from a firstorder lowpass section on the test chip. The circuit is that shown in Fig. 3, with a capacitor value of about $1 \mathrm{pF}$, a bias current ratio $I_{a}=2 I_{b}$ and bias currents generated by applying a voltage to the gate of a (cascoded) nMOS transistor in weak inversion. The gate voltages were linearly spaced, which ideally should yield exponentially-spaced corner frequencies. The data show a falloff of approximately $22 \mathrm{~dB}$ per decade and confirm the exponential spacing of corner frequencies. The response maps well over the entire audio frequency range, although the dynamic range is limited by an approximately $50 \mathrm{~dB}$ noise floor. Note that the transfer function at $100 \mathrm{~Hz}$ corner frequency implies that with the use of base compensation, the bipolar transistors maintain a well-defined exponential I-V relationship even at collector currents as low as $30 \mathrm{pA}$.

The remaining data were measured from the filterbank system chip, the characteristics of which are summarized in Table 1. All measurements shown were made with a power supply of $5 \mathrm{~V}$, though the system showed similar characteristics at $6 \mathrm{~V}$ and $4 \mathrm{~V}$.

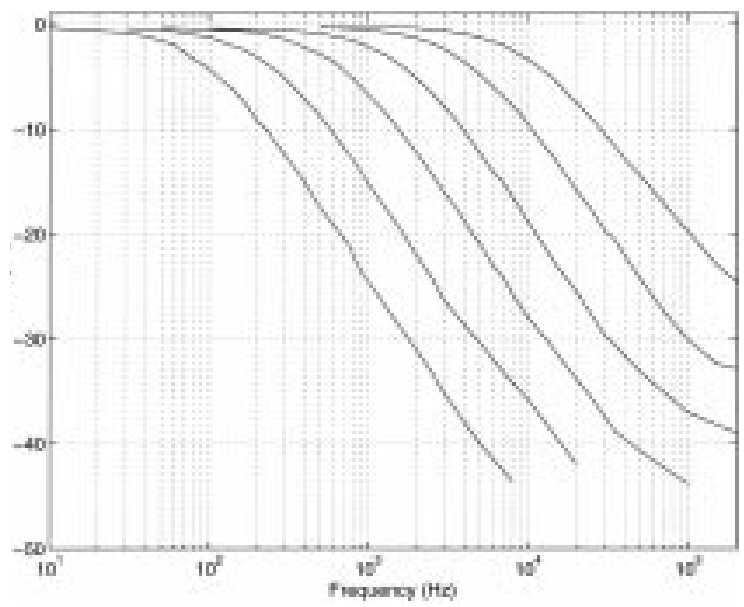

Fig. 11. Measured magnitude response of the log-domain firstorder lowpass filter from the test chip. 
Table 1. Measured filterbank characteristics.

\begin{tabular}{ll}
\hline Die size & $2.2 \mathrm{~mm} \times 2.2 \mathrm{~mm}$ \\
Process & $1.2 \mu \mathrm{m} n$-well BiCMOS \\
& double-poly, double-metal \\
& $4.0-6.0 \mathrm{~V}$ \\
Power supply $V_{d d}-V_{s s}$ & $-0.5 \mathrm{~V}$ \\
$V_{s s}$ & 15 \\
Number of channels & $75 \mu \mathrm{m} \times 234 \mu \mathrm{m}$ \\
Dimensions of bandpass filter & $2.0 \mathrm{pF}$ \\
Capacitor size & $10 \mu \mathrm{m}$ \\
Input DC bias & $10 \mu \mathrm{m}$ \\
Output bias $I_{\mathrm{DC}}$ & $8 \mu \mathrm{A}$ peak-peak. \\
Max. input AC signal & $200 \mu \mathrm{W}$ at $5.0 \mathrm{~V}$ \\
Power consumption & $35 \mathrm{~dB}$ at listed conditions \\
Dynamic range & $30 \mathrm{pA}$ to $3 \mathrm{nA}$ \\
Bias current $I_{b}$ & $50 \mathrm{~Hz}$ to $15 \mathrm{kHz}$ \\
Frequency tuning range & 1 to 10 \\
$Q$ tuning range & $18 \%$ at $Q=6$ \\
Gain nonuniformity across channels & \\
\hline
\end{tabular}

Cascoded MOS transistors implementing the current sources and mirrors plus the $p-n$ junction voltage drops in the translinear loops limit circuit operation at less than approximately $4 \mathrm{~V}$. Power consumption of the circuit is dominated by the DC bias added to the filter input and output, which affects the SNR at the filter output. For the $5 \mathrm{~V}$ power supply and an input/ output bias of $10 \mu \mathrm{A}$, the filter circuit of Fig. 9 dissipates about $200 \mu \mathrm{W}$ and has a dynamic range of about $35 \mathrm{~dB}$.

Fig. 12 shows characteristics of the bandpass filter at three different frequencies in the audio band and



Fig. 12. Measured magnitude response of one bandpass channel in the filterbank system, made of two cascaded second-order logdomain bandpass filters, over three tunings of the center frequency. three different $Q$ values. The filter consists of two second-order bandpass sections of the type shown in Fig. 9, connected in cascade. Both filters in the cascade have adjustable $Q$ values; for our measurements we varied the $Q$ of the first filter while keeping the $Q$ of the second filter fixed. Both filters are biased to have the same peak frequency. Results show that considerable reduction in rolloff occurs on the lower side of the response, due to mismatch of components in the circuit, in particular the matching of bias currents $I_{b}$. These data confirm the simulation results of [8], although it is important to note that the effective gain $(Q)$ of the filter appears to be completely independent of the center frequency (bias current $I_{b}$ ). The full tuning range of the circuit is from about $50 \mathrm{~Hz}$ to $15 \mathrm{kHz}$, limited on the low end by $1 / f$ and thermal noise and on the top end by voltage drops across the CMOS current sources, which were sized appropriately for audio-frequency operation.

The distribution of $Q$ values, listed in Table 1 , shows considerable variation across all channels in the filterbank with the same $V_{Q}$ (see Fig. 9) applied to each. The variation is due primarily to error in matching $I_{b}$ to $I_{b}+(1 / Q) I_{b}$ in Fig. 9. The dependency on $1 / Q$ results in increasingly large gain differences between channels as $Q$ becomes larger. A filterbank with sufficient number of channels cannot necessarily utilize precision trimming of current sources separately for each channel. Although much of the mismatch can be attributed to the quality of the



Fig. 13. Measured center frequencies of all bandpass channels in the filterbank system (circles), compared to the ideal exponential spacing (solid line). 
fabrication process, better matching should be sought through circuit designs which minimize the number of matched current sources, especially those which must match a pMOS current source to an nMOS current sink.

We achieve logarithmic spacing of the channel center frequencies by tapping off a long resistor at equal intervals to get linearly-spaced $V\left(I_{b}\right)$ across the channels. The $I_{b}$ is small enough that the pMOS transistors gated by $V\left(I_{b}\right)$ are in weak inversion, resulting in the nearly perfect spacing seen in Fig. 13.

\section{Conclusions}

We have described how first-order systems can be synthesized from static and dynamic translinear principles, and how to generate higher-order filter transfer functions from these first-order building blocks. In particular, we have addressed circuit design issues relating to audio-frequency applications. We used these synthesis methods to design and fabricate VLSI analog signal processing systems of log-domain filters with current-domain input and output. Results from a first-order lowpass filter and a second-order bandpass filter fabricated in standard BiCMOS technologies show that these filters have transfer functions which map consistently over the entire audio frequency band. Design considerations for low-frequency operation ensure correct translinear operation of the bipolar transistors even at collector currents lower than $10 \mathrm{pA}$. We have characterized performance of the bandpass filters in the context of a high layout density 15-channel filterbank system. Performance of these filters is adequate for the applications for which they were designed [13].

\section{References}

1. Y. Tsividis, "Externally Linear, Time-Invariant Systems and Their Application to Companding Signal Processors." IEEE Trans. Circuits Syst. II 44(2), pp. 65-85, 1997.

2. E. Seevinck, "Companding current-mode integrator: A new circuit principle for continuous-time monolithic filters.', Electron. Lett. 26, pp. 2046-2047, 1990.

3. D. Frey, "Log Domain Filtering for RF Applications.', IEEE J. Solid-State Circuits 31(10), pp. 1468-1475, 1996.

4. J. Mulder, A. van der Woerd, W. Serdijn, and A. Roermund, "General Current-Mode Analysis Method for Translinear Filters.', IEEE Trans. Circuits Syst. I 44(3), pp. 193-197, 1997.

5. E. M. Drakakis, A. Payne, and C. Toumazou, "Log-domain filters, translinear circuits and the Bernoulli Cell.' Proc. ISCAS '97 1, pp. 501-504, 1997.

6. M. Punzenberger and C. Enz, "A new 1.2V BiCMOS Logdomain integrator for companding current mode filters.' $P$ Proc. ISCAS '96 1, pp. 125-128, 1996.

7. D. Perry and G. Roberts, "The Design of Log-Domain Filters Based on the Operational Simulation of LC Ladders.' IEEE Trans. Circuits Syst. II 43(11), pp. 763-774, 1996.

8. R. Fox, M. Nagarajan, and J. Harris, "Practical Design of Single-Ended Log-Domain Filter Circuits.' Proc. ISCAS '97 1, pp. 341-344, 1997.

9. B. Gilbert, "Translinear circuits: a proposed classification." Electron. Lett. 11, pp. 14-16, 1975.

10. E. Vittoz, "Analog VLSI signal processing: why, where, and how?" Analog Integrated Circuits and Signal Processing 6, pp. 27-44, 1994.

11. C. Toumazou, J. Ngarmnill, and T. S. Lande, "Micropower Log-Domain Filter for Electronic Cochlea.', Electronic Letters 30(22), pp. 1839-1841, 1994.

12. W. Serdijn, M. Broest, J. Mulder, A. van der Woerd, and A. van Roermund, "Low-voltage Ultra-low-power Translinear Integrator for Audio Filter Applications.' IEEE Journal of Solid State Circuits 32(4), pp. 577-581, 1997.

13. R. T. Edwards, G. Cauwenberghs, and F. J. Pineda, " A MixedSignal Correlator for Acoustic Transient Classification."' Proc. ISCAS '97 1, pp. 621-625, 1997.



Tim Edwards received his BSEE degree from Duke University, 1990, the MSEE from Stanford University in 1992, and the Ph.D. degree at Johns Hopkins University in 1999. He is currently employed by the Johns Hopkins University Applied Physics Laboratory.

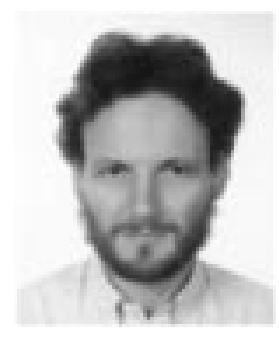

Gert Cauwenberghs received his engineer's degree in applied physics from the University of 
Brussels, Belgium, in 1988, and M.S. and Ph.D. degrees in electrical engineering from the California Institute of Technology in 1989 and 1994, respectively. In 1994, he joined Johns Hopkins University as an assistant professor in electrical and computer engineering. His research covers analog and digital VLSI circuits, systems and algorithms for parallel signal processing and adaptive neural computation. He received the National Science Foundation Career Award in 1997. 